\title{
Techniques for Consecutive TEM and Atom Probe Tomography Analysis of Nanowires
}

D. R. Diercks, ${ }^{*}$ B. P. Gorman, ${ }^{* *}$ C.L. Cheung, ${ }^{* * *}$ G. Wang ${ }^{* * *}$

* Center for Advanced Research and Technology, University of North Texas, Denton, TX 76203

** Department of Metallurgical and Materials Engineering, Colorado School of Mines, Golden, CO 80401

*** Department of Chemistry, University of Nebraska-Lincoln, Lincoln, NE 68588

Nanowires show great promise for development in many technological applications including electronics, photonics, and displays [1-2]. Due to the fine scale of nanowires, transmission electron microscopy (TEM) and atom probe tomography (APT) are among a limited number of techniques that can measure the crystallographic and chemical nature of these structures which ultimately define their performance.

Individual nanowires have a nearly ideal geometry for (APT) analysis. When nanowires are synthesized with the proper length, orientation, and inter-wire separation spacings, nanowire APT analysis is relatively straightforward and has yielded reasonable results [3]. However, for many nanowire growth processes, it is difficult or undesirable to grow structures with these restrictions. One preparation technique demonstrated for APT analysis in such circumstances is a protected liftout method [4]. This research demonstrates two other methods for analysis of individual nanowires by APT. Previously developed techniques have demonstrated cross-correlative TEM and APT analysis on focused ion beam (FIB) prepared lift-out samples [5]. Performing TEM analysis of the sample before atom probe analysis is useful not only for obtaining complementary information about the crystal structure(s), but also for assisting in accuracy of the reconstruction. The radius, shank angle, layer thicknesses, and other structural information can be directly measured and incorporated into the atom probe reconstruction. This work expands the previously reported technique to both dispersed and substrate grown nanowires. This technique as applied to nanowires utilizes only the electron beam in order to avoid structural damage from the FIB column.

In the first adaptation, the nanowires were removed from their substrate, suspended in ethanol, and then dispersed over a porous substrate. This geometry allows most of the nanowires to be retained on the surface suspended over the pore openings (Figure 1a). A nano-manipulator was placed in close proximity to the nanowire's base. A very small electron beam deposited platinum (e-beam Pt) weld was made at that location to provide just enough attachment to remove the wire from the porous substrate (Figure 1b). The nanowire was then moved to a TEM grid that had been sectioned such that a vertical portion of each grid bar extended beyond the horizontal grid bar. The nanowire was aligned with a vertical grid bar and securely welded with e-beam Pt. The nano-manipulator was then slowly drawn away from the nanowire until the initial small weld detached, leaving the nanowire mounted to the TEM half-grid (Figures 1c and 1d).

In the second adaptation, the nanowires were left attached to the substrate on which they were grown. That substrate was mounted orthogonal to the nano-manipulator such that the nanowires were parallel with the nano-manipulator tip. A nanowire was securely welded, again using e-beam $\mathrm{Pt}$, to the nano-manipulator which was then pulled away from the substrate to detach the nanowire (Figure 2). Omniprobe's Short $\mathrm{Cut}^{\mathrm{TM}}$ technique was then used to simultaneously section and embed 
the nano-manipulator needle into a TEM half-grid for TEM and APT analysis as described in reference [5].

These described techniques were used to analyze $\mathrm{NdB}_{6}, \mathrm{ZnO}$, and $\mathrm{Ge}$ nanowires and were performed on an FEI Nova 200 dual beam system equipped with an Omniprobe Autoprobe 250. (The FIB column was not used). TEM analysis was completed on an FEI Tecnai F20 S-Twin. The atom probe analysis was performed on an Imago Scientific Instruments LEAP 3000X using both voltage and laser pulsed evaporation. Some of the hardware used was designed by Hummingbird Scientific Instruments and Omniprobe, Inc. [5].

\section{References}

[1] M. S. Gudiksen et al., Nature, 415, (2002) 617.

[2] C. J. Lee et al., Appl. Phys. Lett., 81 (2002) 3648.

[3] D. E. Perea et al., Nano Lett., 6 (2006) 181.

[4] T. J. Prosa et al., Microsc. Microanal., 14(Suppl 2), (2008) 456.

[5] B. P. Gorman et al., Microsc. Today, 16 (2008) 42.

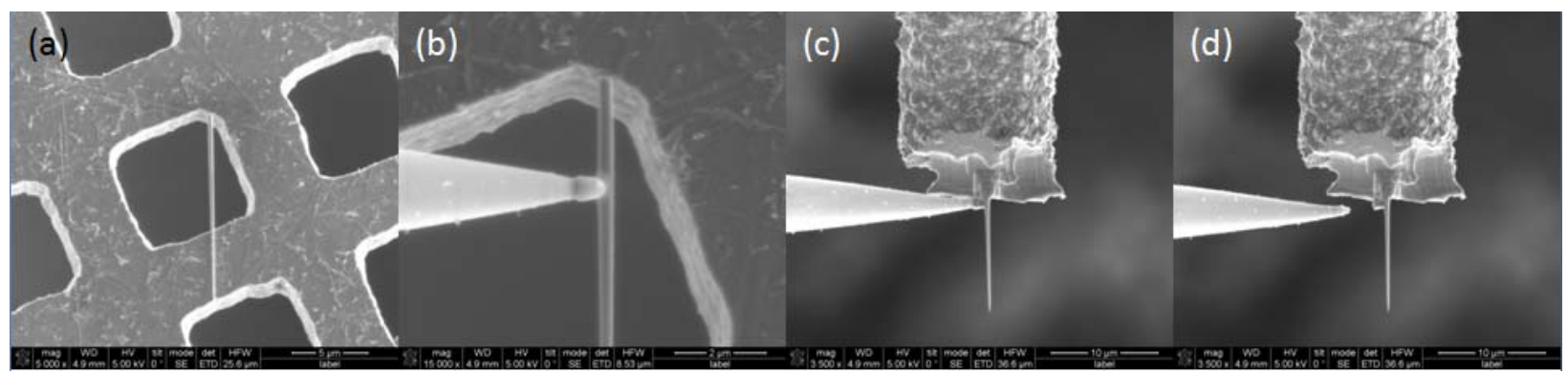

Figure 1 - Mounting of a nanowire dispersed on a porous substrate to a half-TEM grid post for TEM and APT analysis.

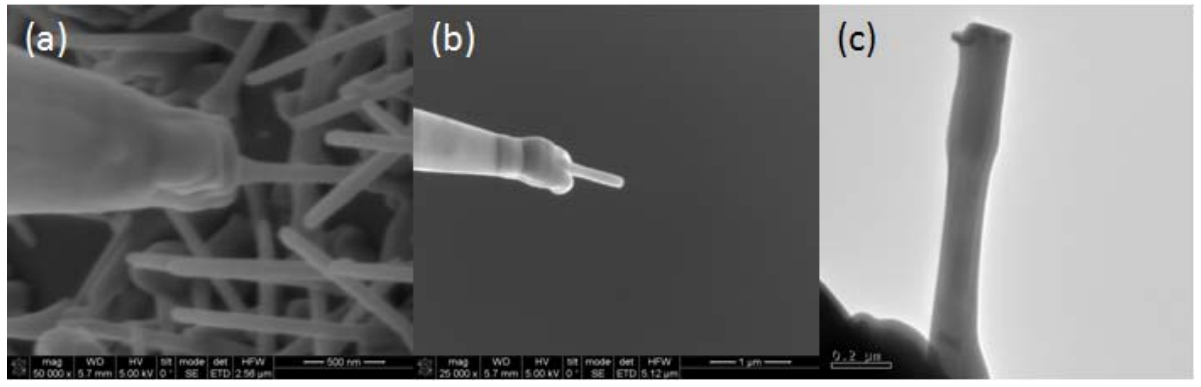

Figure 2 - (a) Attachment of the nano-manipulator to a nanowire and (b) removal of the nanowire directly from the substrate. (c) TEM image of the nanowire.

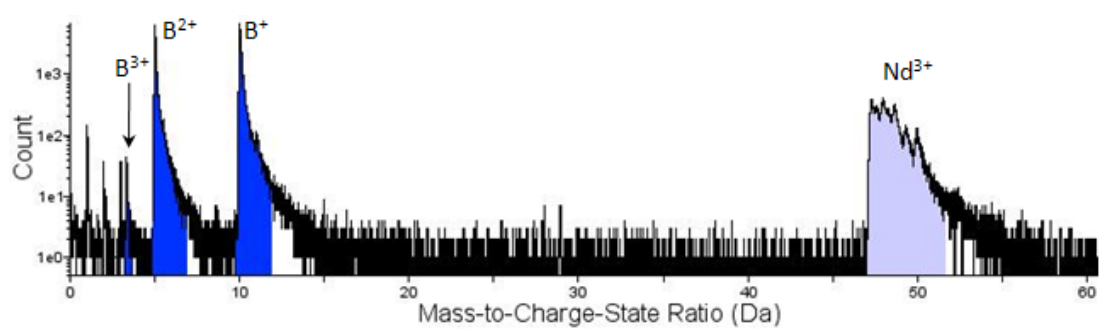

Figure 3 - Mass spectrum from atom probe analysis of a $\mathrm{NdB}_{6}$ nanowire. 